Apidologie, 1979, 10 (4), 341-358.

\title{
RÔLE DE L'ACTIVITÉ DE L'EAU DANS LA CRISTALLISATION DU MIEL
}

\section{Über die Rolle der Gleichgewichtsfeuchtigkeit $\mathrm{a}_{\mathrm{w}}$ (Wasseraktivität) bei der Kristallisation des Honigs}

\author{
T. TABOURET \\ Laboratoire de recherches sur la Réactivité des Solides \\ Faculté des Sciences Mirande, B.P. 138 \\ 21004 Dijon Cedex
}

\begin{abstract}
SUMMARY
THE INFLUENCE OF WATER ACTIVITY ON HONEY CRYSTALLIZATION
\end{abstract}

J. W. WHITE's factor $\frac{D}{W}(1962)$ does not prove satisfactory in accounting for honey tendency to crystallize especially as regards low humidity honeys. The present investigation concerns such low humidity honeys and shows that their water activity is of utmost importance in their crystallization. Further it is pointed out that in a great number of cases, the water activity influence must be taken into account to explain or to predict the crystallization behaviour of usuel honeys.

\section{RÉSUMÉ}

L'indice de J. W. WHITE (1962) $\frac{D}{W}$ se montre peu satisfaisant pour rendre compte de la tendance des miels à cristalliser, particulièrement les miels de faible humidité. Le travail présenté ici concerne ces mielsci et montre que l'activité de leur eau est de la plus grande importance pour leur cristallisation. On fait en outre remarquer qu'il faut, dans un grand nombre de cas, tenir compte de l'influence de l'activité de l'eau pour expliquer ou prédire le comportement de cristallisation des miels courants. 


\section{INTRODUCTION}

Depuis plus de cinquante ans, les chercheurs qui s'intéressent aux miels ont essayé de prédire leur tendance à cristalliser d'après leur composition chimique. En effet, en dehors de tout traitement technologique, certains miels, tel le miel de colza (Brassica napus, var. oleifera), cristallisent en peu de jours. D'autres cristallisent au bout de quelques mois. D'autres encore, comme les miels de sauge (Salvia sp.) ou d'acacia (Robinia pseudacacia) ne cristallisent jamais, même après plusieurs années de conservation.

La pasteurisation retarde la cristallisation des miels qui ont une tendance naturelle à cristalliser mais elle ne peut l'empêcher définitivement (GoNNET, 1977).

On admet généralement à la suite des travaux de J. W. WhITE et al. (1962) qu'il existe une corrélation satisfaisante entre la tendance des miels à cristalliser et le rapport $\frac{\text { Glucose }}{\text { Eau }}=\frac{D}{W}$ et l'on attribue aux différents échantillons une " note de cristallisation " comprise entre 0 et 9 , proportionnelle à leur degré de cristallisation que l'on apprécie visuellement (Tabl. 1).

TABL. 1. - Degré de cristallisation (d'après WhiTE et al., 1962).

TAB. 1. - Kristallisationsgrad (nach WHITE et al., 1962).

\begin{tabular}{|c|c|c|}
\hline $\begin{array}{l}\text { Note } \\
\text { Note }\end{array}$ & $\begin{array}{l}\text { Aspect } \\
\text { Aussehen }\end{array}$ & $D / W$ \\
\hline 0 & $\begin{array}{l}\text { Entièrement liquide } \\
\text { Klarflüssig }\end{array}$ & 1,58 \\
\hline 1 & $\begin{array}{l}\text { Quelques cristaux epars } \\
\text { Vereinzelte Kristalle }\end{array}$ & 1,76 \\
\hline 2 & $\begin{array}{l}\text { Couche cristallisée sur une hauteur de } 1,5 \text { à } 3 \mathrm{~mm} \\
\text { Kristallschicht } 1,5-3 \mathrm{~mm} \text { hoch }\end{array}$ & 1,79 \\
\hline 3 & $\begin{array}{l}\text { Quelques amas cristallins } \\
\text { Einige Kristallansammlungen }\end{array}$ & 1,86 \\
\hline 4 & $\begin{array}{l}\text { Couche cristallisée sur une hauteur de } 6 \text { à } 12 \mathrm{~mm} \\
\text { Kristallschicht } 6-12 \mathrm{~mm} \text { hoch }\end{array}$ & 1,83 \\
\hline 5 & $\begin{array}{l}\text { Echantillon cristallise sur } 1 / 4 \text { de la hauteur du miel } \\
\text { Honigprobe bis zu einem Viertel der Höhe kristallisiert }\end{array}$ & 1,99 \\
\hline 6 & $\begin{array}{l}\text { Échantillon cristallisé sur } 1 / 2 \text { de la hauteur de miel } \\
\text { Honigprobe bis zur halben Höhe kristallisiert }\end{array}$ & 1,98 \\
\hline 7 & $\begin{array}{l}\text { Echantillon cristallise sur } 3 / 4 \text { de la hauteur de miel } \\
\text { Honigprobe bis zu drei Vierteln der Höhe kristallisiert }\end{array}$ & 2,06 \\
\hline 8 & $\begin{array}{l}\text { Échantillon entièrement cristallisé mou (aspect solide ou trés visqueux) avec éventuelle- } \\
\text { ment une mince couche liquide surnageant }(1-3 \mathrm{~mm} \text { ) } \\
\text { Ganze Probe weich kristallisiert (fest oder sehr dickflüssig) gelegentlich mit } 1.3 \mathrm{~mm} \text { überstelkender } \\
\text { Flüssigkeit }\end{array}$ & 2,16 \\
\hline 9 & $\begin{array}{l}\text { Échantillon entièrement cristallisé dur } \\
\text { Ganze Probe hart kristallisiert }\end{array}$ & 2,24 \\
\hline
\end{tabular}


Mais cette corrélation est contestée: SIDDIQUi (1970), HADORN et ZÜRCHER (1974). Par ailleurs, en examinant les données statistiques fournies par J. W. WHITE et ses collaborateurs, on est frappé par deux faits :

$1^{\circ}$ Rares sont les miels dont la teneur en eau, que nous désignerons désormais par " $W$ ", est inférieure à $15 \mathrm{p}$. cent et très rares ceux pour lesquels $W$ est inférieure à $14 \mathrm{p}$. cent : il n'y en a que 4 ou 5 sur 504 échantillons examinés au total par ces auteurs (voir Tabl. 6).

$2^{\circ}$ Les miels peu humides ont en général une note de cristallisation bien inférieure à celle qui est prévisible d'après le rapport $\frac{D}{W^{\prime}}$ C'est le cas de tous les échantillons où $W$ est inférieure à $14 \mathrm{p}$. cent qui devraient avoir d'après le tableau 1 la note 8 ou 9 et qui ont tous, en fait, reçu la note 1 (J. W. White et al., 1962. Échantillons nos 423,440 , 450 et 493$)$.

Ces constatations nous ont incité à une première étude, celle de la tendance des miels à cristalliser en fonction de $W$, plus particulièrement aux faibles valeurs.

Parmi les auteurs qui ont essayé d'établir une corrélation entre la composition des miels et leur tendance à cristalliser, AuSTIN (1965) est le seul qui ait préconisé de ramener leur composition réelle à une même valeur de $W$, par exemple 17,5 p. cent. Mais, s'il a fait l'unanimité contre lui, rares sont ceux qui ont recherché une corrélation entre $W$ et la tendance à cristalliser. Aucun auteur, à notre connaissance, n'a exploré le domaine des faibles valeurs de $W$.

Fulmer et Bosch (1932, 1934), cités par Jamieson (1954), ont étudié la cristallisation de miels de mélilot (sweet clover, Melilotus sp.) de $W$ comprise entre 17,7 p. cent et 25,9 p. cent, par intervalles de 1 p. cent, pendant quatorze mois. Au-dessus de 20 p. cent d'eau, ces miels ne cristallisent pas, ou très peu.

Jamieson (1954) a étudié quatre miels différents de $W$ allant de $15,1 \mathrm{p}$. cent à 22,1 p. cent pendant douze mois et trouvé des résultats semblables, reproduits dans le tableau 2. On remarque dans ce tableau l'existence de maxima de cristallisation en fonction de la teneur en eau croissante, donnant à penser que, pour un miel donné ramené à des valeurs de $W$ plus basses, la cristallisation peut être plus faible, bien que le rapport $\frac{D}{W}$ soit supérieur.

Codounis (1962) a étudié le comportement de cristallisation de solutions pures de dextrose et de lévulose de concentration variable ( $W$ comprise entre 16 et 30 p. cent). Nous discuterons plus loin l'intérêt de ces études. Le comportement de cristallisation des solutions pures est fort différent de celui du miel; toutefois, les travaux de Codounis confirment ceux de Jamieson (1954) en ce qui concerne le rôle inhibiteur de cristallisation du lévulose (ou fructose). Ce rôle inhibiteur est bien connu et nombre d'auteurs relient la tendance des miels à cristalliser à la concentration en 
TABL. 2. - Volume de cristaux ( $\mathrm{ml}$ ) obtenus par centrifugation de $30 \mathrm{ml}$ d'échantillon de miel après 12 mois de cristallisation (d'après JAMIESON, 1954).

ТАВ. 2. - Volumen der Kristalle $(\mathrm{ml})$, das durch Zentrifugieren einer Honigprobe von $30 \mathrm{ml}$ nach 12 monatiger Kristallisation erhalten wurde (nach JAMIESON, 1954).

\begin{tabular}{|c|c|c|c|c|}
\hline $\begin{array}{l}W \text { pour cent } \\
W \text { prozent }\end{array}$ & $\begin{array}{l}\text { Sweet clover } \\
\text { (Melilot) } \\
\text { ml. } \\
\text { (Honigklee) }\end{array}$ & $\begin{array}{c}\text { White clover } \\
\text { (Trèfle blanc) } \\
\text { ml. } \\
\text { (Weissklee) }\end{array}$ & $\begin{array}{c}\text { Buckwheat } \\
\text { (Sarrasin) } \\
\text { ml. } \\
\text { (Buchweizen) }\end{array}$ & $\begin{array}{c}\text { Fall flower } \\
\text { (Fleurs d"automne) } \\
\text { ml. } \\
\text { (Herbstblüten) }\end{array}$ \\
\hline 15,1 & 18,0 & 1,0 & - & - \\
\hline 16,2 & 19,0 & 1,5 & - & $\ldots$ \\
\hline 17,1 & 18,0 & 1,0 & 11,0 & 1,0 \\
\hline 18,2 & 4,0 & $\begin{array}{l}\text { quelques } \\
\text { cristaux } \\
\text { einige }\end{array}$ & 3,5 & 1,5 \\
\hline 19,3 & 3,5 & $\begin{array}{c}\text { Kristalle } \\
\text { quelques } \\
\text { cristaux } \\
\text { einige } \\
\text { Kristalle }\end{array}$ & 0,5 & 0.5 \\
\hline 20,2 & 1,5 & 0 & 0 & 0 \\
\hline 21,2 & 0,5 & 0 & 0 & 0 \\
\hline 22,1 & 0 & 0 & 0 & 0 \\
\hline
\end{tabular}

lévulose et font intervenir le rapport $\frac{L}{D}$ (lévulose/glucose) ou $\frac{D}{L}$ au lieu de $\frac{D}{W}$. Mais ce rôle inhibiteur reste à l'heure actuelle inexpliqué.

Nous avançons l'hypothèse qu'il est en rapport avec l'activité de l'eau dans le miel $\left(a_{w}\right)$, que l'on identifie à l'humidité relative d'équilibre (HRE ou, en anglais ERH), laquelle est fonction aussi de $W$. Rappelons que l'activité de l'eau se définit comme étant le rapport suivant :

tension de la vapeur d'eau à la surface du produit

$$
\begin{aligned}
& a_{w}=\frac{f_{t}}{F_{t}}=\frac{\text { à la température } t}{\text { tension de la vapeur d'eau à la surface de l'eau libre }} \\
& \text { à la même température } t
\end{aligned}
$$

L'activité de l'eau dans un miel varie donc en sens inverse de l'hygroscopicité de ce miel.

L' $a_{w}$ des miels n'a pas fait l'objet jusqu'à présent de nombreuses déterminations et aucune corrélation n'a jamais été tentée avec leur tendance à cristalliser. CRANE (1975) mentionne seulement dans sa synthèse bibliographique les valeurs de MARTIN (1958).

Nous avons donc déterminé l'a $a_{w}$ des miels de faible teneur en eau objet de notre étude de base (voir page 347). 
D'autre part, pour mieux vérifier l'hypothèse avancée, nous avons calculé de façon approchée l' $\mathrm{a}_{w}$ des miels étudiés par nos prédécesseurs et cherché une corrélation entre celle-ci et leur tendance à cristalliser.

\section{MATÉRIEL ET MÉTHODES}

FABRICATION DE MIELS DE FAIBLE TENEUR EN EAU $(w)$

Nous avons choisi pour nos travaux un miel de colza ayant naturellement une forte tendance à cristalliser. Sa composition est la suivante pour cent :

\begin{tabular}{c|c|c|c|c}
\hline$W$ & $L$ & $D$ & Autres sucres & $D / W$ \\
\hline 16,1 & 37,8 & 39,3 & 5,81 & 2,44 \\
\hline
\end{tabular}

$W$ a pu être ramenée à 8 p. cent par déshydratation sous vide. Nous avons publié récemment les techniques que nous avons mises au point pour les échantillons de laboratoire (TABOURET, 1977)et pour les traitements à l'échelle industrielle (BORNECK et TABOURET, 1977). Notre technique de laboratoire élimine les cristaux et les germes se trouvant dans le miel à l'origine, à l'égal d'une bonne pasteurisation.

Nous avons ensuite redilué partiellement le miel déshydraté, de manière à réaliser une gamme de $W$. Pour chaque valeur de $W$, deux échantillons ont été mis en pots, dont l'un a été ensemencé au préalable avec quelques cristaux de dextrose hydrate pharmaceutique. Les deux pots ont été ensuite hermétiquement fermés et conservés quatorze mois à température ambiante. Un échantillon supplémentaire à $8 \mathrm{p}$. cent d'eau a été conservé dans les mêmes conditions dans un pot muni d'un couvercle non étanche. Le témoin était constitué par le miel naturel refondu au bain-marie à $45^{\circ} \mathrm{C}$.

\section{MESURE DE L'ACTIVITÉ DE L'EAU $\left(a_{w}\right)$}

La méthode est la même que celle utilisée généralement pour mesurer l' $a_{w}$ des solutions de sucres. Le principe est le suivant : la solution à étudier échange son humidité avec l'air ambiant jusqu'à l'équilibre. Au moyen d'un appareillage approprié on mesure l'humidité relative de l'air à l'équilibre qui n'est autre que l'a $a_{w}$ cherchée. C'est pourquoi on appelle également l'a $a_{w}$ " humidité relative d'équilibre ". En variante, on peut mesurer la teneur en eau $W$ des solutions obtenues après équilibre avec différentes ambiances d'humidité relative connue. Cette variante, préconisée par l'A.S.T.M. (1951-1963), est celle que nous avons utilisée.

\section{CALCUL DE L'ACTIVITÉ DE L'EAU $\left(a_{w}\right)$}

Un calcul approché de l'a $a_{w}$ des miels est possible à partir de la composition en sucres.

L' $a_{w}$ des solutions de sucres a été déterminée par Grover (1947), MONEY et BorN (1951), NorRIsH (1966), LoNCIN et al. (1978). Tous ces auteurs, sauf LoNCIN et al., donnent une méthode de calcul de l'a à partir de la composition de la solution. Nous avons calculé $a_{w}$ à partir de la méthode de Grover. Il nous est apparu que, en dépit de son empirisme, cette méthode est la plus commode et donne les résultats les plus conformes à nos propres résultats expérimentaux. 
Cette méthode permet le calcul de l'a $a_{w}$ des solutions sucrées de confiserie à partir de leur composition. Elle comporte dans une première étape le calcul de l'équivalent saccharose $E_{s}$, qui est la concentration, exprimée en saccharose/eau, de la solution fictive de saccharose pur ayant la même $a_{w}$ que la solution considérée, à l'aide de la formule empirique de Grover (1947) $\mathrm{n}^{\circ} 3$ :

$$
E_{s}=s+1,3 i+0,8 g
$$

où

$s=$ saccharose/eau de la solution considérée,

$i=$ inverti/eau de la solution considérée, c'est-à-dire (glucose + lévulose)/eau,

$g=$ matière sèche de sirop de glucose/eau.

(Le sirop de glucose est essentiellement une solution de glucose et de ses polymères en proportions variables.)

Dans une deuxième étape, à chaque valeur de $E_{s}$ ainsi calculée correspond une valeur de $a_{k^{\prime}}$ selon un tableau établi expérimentalement une fois pour toutes par Grover (1947), tableau dont les valeurs peuvent se calculer par la formule empirique de Grover (1947) $\mathrm{n}^{0} 4$ :

$$
100 a_{w}=104-10 E_{s}+0,45 E_{s}^{2}
$$

Pour transposer cette méthode au cas des miels, nous utilisons la formule (3) et le tableau de Grover (1947) en posant :

et

$$
\begin{gathered}
i=\frac{\mathrm{L}+D}{W} \\
g=\frac{100-W-(L+D)}{W}
\end{gathered}
$$

où

$L=$ pour cent lévulose du miel,

$D=$ pour cent glucose du miel,

$W=$ pour cent d'eau du miel.

Par suite $s=0$.

Nous justifions ces approximations de la façon suivante :

La formule (3) montre que, grosso modo, si l'on attribue à une solution de saccharose l'hygroscopicité 1 , celie de la solution d'inverti de même concentration est 1,3 tandis que celle du sirop de glucose de même concentration n'est que de 0,8 .

Dans la majorité des miels, la teneur en lévulose est supérieure à la teneur en glucose : $L>D$. Il ne serait pas rationnel de poser $i=\frac{2 D}{W}$, car le lévulose « en excès » $\frac{L-D}{W}$ ne serait pas représenté dans la formule (3), ni en l'assimilant à $s$, ni en l'assimilant à g. Le lévulose, en effet, est plus hygroscopique que le saccharose et que le glucose, HeIss (1968), LoNCIN et al. (1978). L'approximation (1) revient à attribuer au lévulose " en excès " $(L-D)$ la même hygroscopicité que celle de l'inverti. L'approximation (2) revient à assimiler l'hygroscopicité globale des autres solutés des miels (y compris le saccharose) à celle du sirop de glucose. Elle se justifie compte tenu de la similitude de composition entre ces produits.

\section{RÉSULTATS}

Cristallisation du miel de colza déshydraté

Les résultats sont rassemblés dans le tableau 3. Tous les échantillons dont $W$ est inférieure à 14 p. cent et qui ne sont pas ensemencés restent parfaitement liquides après 
TABL. 3. - Cristallisation du miel de colza déshydraté

(On a utilisé la notation de WhITE et al. - Voir Tabl. 1).

ТАВ. 3. - Kristallisation von entwässertem Rapshonig

(Wir benutzten die Benotung nach WhITE et al. - Siehe Tab. 1)

\begin{tabular}{|c|c|c|c|c|c|}
\hline \multirow{2}{*}{$\begin{array}{l}W \text { pour cent } \\
W \text { Prozent }\end{array}$} & \multirow{2}{*}{$\begin{array}{l}\text { Échantillon } \\
\mathrm{NE}=\text { non ensemencé } \\
\mathrm{E}=\text { ensemencé } \\
\text { Probe } \\
\mathrm{NE}=\text { nicht angeimpft } \\
\mathrm{E}=\text { angeimpft }\end{array}$} & \multicolumn{3}{|c|}{$\begin{array}{c}\text { Cristallisation après } \\
\text { Kristallisation nach }\end{array}$} & \multirow{2}{*}{$D / W$} \\
\hline & & $\begin{array}{l}1 \text { mois } \\
1 \text { Monat }\end{array}$ & $\begin{array}{l}31 / 2 \text { mois } \\
31 / 2 \text { Monaten }\end{array}$ & $\begin{array}{l}14 \text { mois } \\
14 \text { Monaten }\end{array}$ & \\
\hline \multirow[t]{2}{*}{8,0} & $\mathrm{NE}$ & 0 & 0 & 0 & 5,39 \\
\hline & E & $0+$ & 1 & 2 & \\
\hline \multirow[t]{2}{*}{10,0} & $\mathrm{NE}$ & 0 & 0 & 0 & 4,22 \\
\hline & $E$ & $0+$ & 1 & 2 & \\
\hline 12,1 & $E$ & $0+$ & 2 & 2 a 3 & 3,40 \\
\hline 13,6 & $\mathrm{NE}$ & 0 & 0 & 0 & 2,98 \\
\hline \multirow[t]{2}{*}{14,5} & NE & 0 & 1 & 2 a 3 & 2,76 \\
\hline & $\mathrm{E}$ & 0 & 3 & 5 a 6 & \\
\hline \multirow[t]{2}{*}{15,9} & $\mathrm{NE}$ & 0 & 2 & 5 & 2,48 \\
\hline & $E$ & 6 & 8 & 9 & \\
\hline 16,1 & Témoin & 4 & 6 & 9 & 2,44 \\
\hline 8,0 & $\begin{array}{l}\text { Kontrolle } \\
\text { NE couvert non étanche } \\
\text { NE Deckel nicht dicht }\end{array}$ & 0 & 0 & 1 & $5,39\left(^{*}\right)$ \\
\hline
\end{tabular}

$\left(^{*}\right)$ Après 14 mois de conservation en atmosphère très humide $W=24,2$.

Nach 14 monatiger Lagerung in sehr feuchter Atmospäre $W=24,2$.

treize mois de conservation à température ambiante, même lorsque le pot n'est pas fermé de façon étanche. Même lorsqu'ils sont ensemencés, ces échantillons ne cristallisent que très lentement, plus lentement que les échantillons non ensemencés mais plus riches en eau.

Nous avons également constaté que le miel déshydraté par le procédé CENTRITHERM (BORNECK et TABOURET, 1977), c'est-à-dire par chauffage sous vide quelques secondes à $55^{\circ} \mathrm{C}$ seulement, donc sans effet de pasteurisation, ne cristallise que très lentement. La déshydratation partielle vient donc renforcer la pasteurisation dans la prévention de la cristallisation. La limite critique de $W$ semble se situer, pour ce type de miel, à 13 ou 14 p. cent environ.

\section{Détermination de l'a $\mathrm{a}_{\mathrm{w}}$ du miel de colza déshydraté}

Nous avons mesuré par la variante de la méthode décrite (page 345) l' $\mathrm{a}_{w}$ du miel de colza ayant servi à nos expériences et parallèlement celle d'une solution de même composition initiale en dextrose, lévulose et eau, soit :

$$
\text { Eau }=16,1 \quad D=39,3 \quad L=37,8 \quad \text { Total }=93,2
$$


Nous avons dédoublé chaque échantillon en :

- un échantillon non pasteurisé, marqué NP;

- un échantillon pasteurisé $30 \mathrm{mn}$ à $65^{\circ} \mathrm{C}$, marqué $\mathrm{P}$.

Nos résultats sont consignés dans le tableau 4.

TABL. 4. - W pour cent à l'équilibre du miel de colza el de la solution de même $\mathrm{D}$ et $\mathrm{L}$, en fonction de $\mathrm{a}_{w}$.

ТАв. 4. - W Prozent beim Gleichgewichtszutand zwischen Rapshonig und einer Lösung mit gleichem $\mathrm{D}$ und $\mathrm{L}$, Wert in Abhängigkeit l'on $\mathrm{a}_{\boldsymbol{w}}$. $\mathrm{NP}=$ nicht pasteurisiert; $\mathrm{P}=30 \mathrm{Min}$. bei $65^{\circ} \mathrm{C}$ pasteurisiert.

\begin{tabular}{|c|c|c|c|c|c|c|c|}
\hline $\begin{array}{l}W \text { pour cent } \\
\text { a l'équilibre } \\
W \text { Prozent im } \\
\text { Gleichgewichtszutand }\end{array}$ & 1 & 0,9 I & 0,75 & 0,625 & 0,46 & 0,29 & 0,20 \\
\hline $\begin{array}{l}\text { Miel NP } \\
\text { Honig NP }\end{array}$ & 44,6 & 40,0 & 26,8 & 17,1 & 11,0 & 8,6 & 7,6 \\
\hline $\begin{array}{l}\text { Miel P } \\
\text { Honig I }\end{array}$ & 44,4 & 39,8 & 26,8 & 17,1 & 11,1 & 8,6 & \\
\hline $\begin{array}{l}\text { Solution NP } \\
\text { Lösung NP }\end{array}$ & 59,0 & 40,9 & & 17,7 & 11,8 & 9,6 & \\
\hline $\begin{array}{l}\text { Solution P } \\
\text { Lösung P }\end{array}$ & 58,2 & 41,2 & & 17.7 & 11,3 & 10,0 & \\
\hline
\end{tabular}

La pasteurisation ne semble pas influencer l' $a_{w}$ de façon significative. La solution a une teneur en eau d'équilibre supérieure à celle du miel, à $a_{n}$ égale, ce qui signifie qu'elle est plus hygroscopique que le miel, surtout aux $W$ élevées. Mais on voit qu'on peut, en première approximation, assimiler l'a $a_{w}$ du miel à celle de la solution. Or, l' $a_{w}$ de la solution peut être calculée à partir de la composition.

Sur la figure 1 , nous avons reporté les résultats de différentes sources. Ce tracé a permis de déterminer les $a_{w}$ des échantillons déshydratés. Pour les autres miels, nous avons calculé $a_{w}$ par la méthode de Grover.

Dans le tableau 5 sont mentionnés, pour chaque miel déshydraté (et réhydraté) le rapport $D / W$ (Voir Tabl. 3) et l' $a_{w}$. On voit que l' $a_{w}$ diminue avec $W$ du miel tandis que $D / W$ augmente, jusqu'à atteindre des valeurs très basses excluant toute cristallisation, même avec ensemencement. Nous concluons donc que dans la zone considérée des valeurs de $W, a_{w}$ a plus d'influence sur la vitesse de cristallisation que $D / W$.

\section{DISCUSSION}

Si l'on se reporte aux valeurs de $D / W$ des échantillons déshydratés (Voir Tab. 3), il est clair que plus ce rapport est élevé, moins le miel a tendance à cristalliser, ce qui 


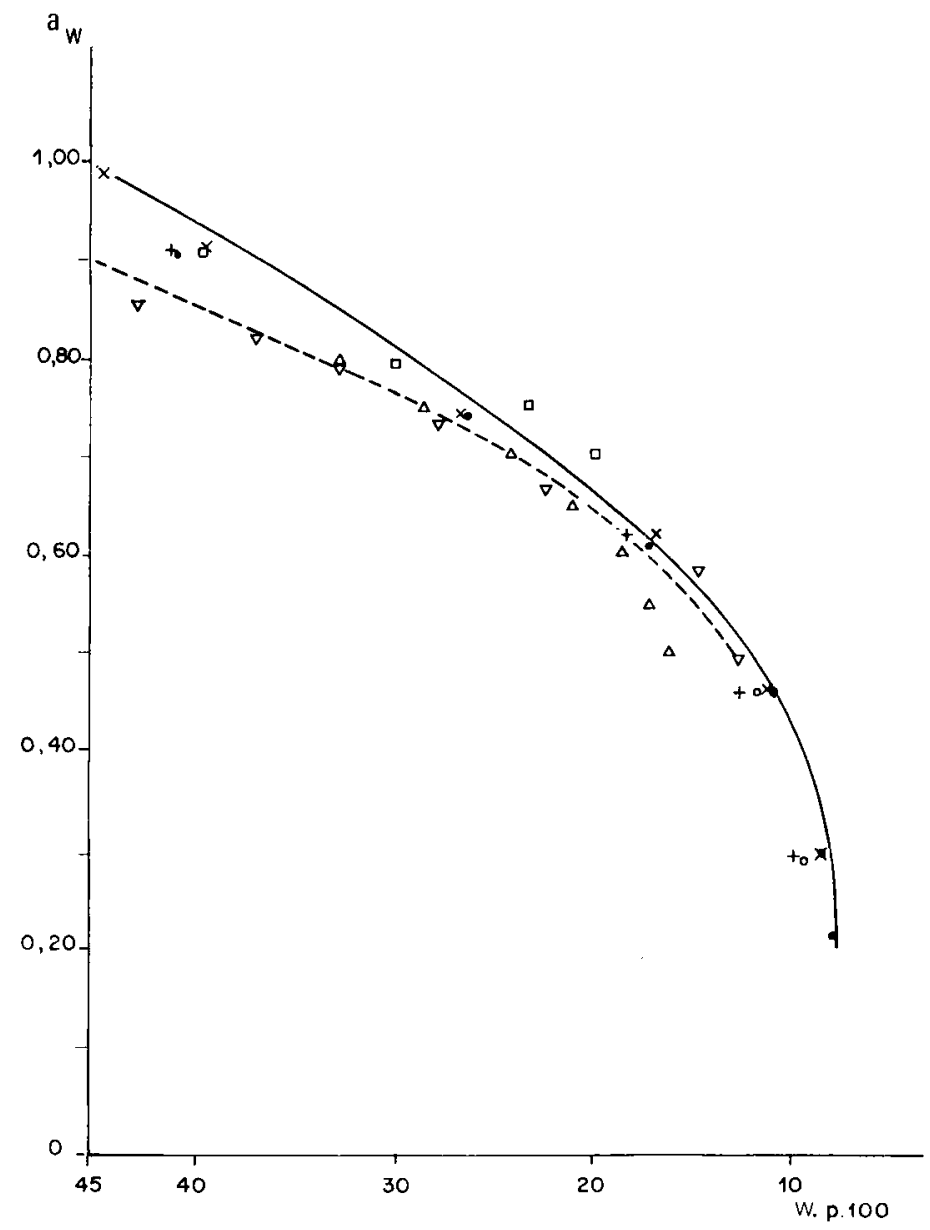

FIG. 1. - Activité de l'eau $\mathrm{a}_{\mathrm{w}}$ (humidité relative d'équilibre) du miel et de produits sucrés en fonction de leur teneur en eau $W$.

- miel de colza non pasteurisé

$x$ miel de colza pasteurisé

o solution similaire non pasteurisée

+ solution similaire pasteurisée

$\triangle$ miel de trèfle, d'après MARTIN

$\square$ miel artificiel d'après HeIss, 1968

$\nabla$ inverti d'après Grover, 1947

Aвв. - Wasseraktivität $\mathrm{a}_{\mathrm{w}}$ (relative Gleichgewichtsfeuchtigkeit)

von Honig und Zuckerprodukten in Abhängigkeit von ihrem Wassergehalt (W)

- nicht pateurisierter Rapshonig

$\left.\begin{array}{l}\times \text { pasteurisierter } \text { Rapshonig } \\ O \text { vergleichbare, nicht pasteurisierte Lösung } \\ + \text { vergleichbare pasteurisierte Lösung }\end{array}\right\}$ siehe Tabelle 4

+ vergleichbare pasteurisierte Lösung

$\triangle$ Kleehonig nach MARTIN

$\square$ Kunsthonig nach HEISs, 1968

$\nabla$ Invertzucker nach GROVER, 1947 
TABL. 5. - Comparaison des miels de colza déshydratés.

TAB. 5. - Vergleich der entwässerten Rapshonige.

\begin{tabular}{|c|c|c|c|c|c|c|c|}
\hline & \multicolumn{7}{|c|}{$\begin{array}{c}W \text { du miel de colza déshydraté (en pour cent) } \\
W \text { des entwässerten Rapshonigs (in Prozent) }\end{array}$} \\
\hline & 8.0 & 10.0 & 12,1 & 13,6 & 14.5 & 15.9 & $\begin{array}{l}16,1 \\
\text { témoin non } \\
\text { déshydraté } \\
\text { nicht entwässerte } \\
\text { Kontrolle }\end{array}$ \\
\hline$D / W(1)$ & 5,39 & 4,22 & 3,40 & 2,98 & 2,76 & 2,48 & 2,44 \\
\hline $\begin{array}{l}a_{w} \text { expérimentale } \\
\text { experimentelle } a_{w}\end{array}$ & $\leqslant 0,25$ & 0.35 & 0,47 & 0,53 & 0.56 & 0.60 & 0,60 \\
\hline$I_{2}=\frac{D / W}{\left(1-a_{w}\right)^{2}}$ & $\leqslant 9,58$ & 9.98 & 12,11 & 13,472 & 14.266 & 15,481 & 15,256 \\
\hline $\begin{array}{l}\text { Note de cristallisation selon WHITE } \\
\text { et al., } 1962(2) \\
\text { Benotung der Kristallisation nach WHITE } \\
\text { et al., } 1962(2)\end{array}$ & 0 & 0 & $2(3)$ & 0 & 2 a 3 & 5 & 9 \\
\hline
\end{tabular}

(1) D est calculé selon la formule : $D=39.3 \times \frac{(100-W)}{83,9}, 39,3$ et 83.9 ćtant respectivement le glucose et le Brix du temoin.

(2) Échantillons non ensemencés conservés 14 mois à température ambiante.

(3) Échantillon ensemence avant conservation.

(1) D ist berechnet nach der Formel $D \cdot 39.3 \times \frac{(100-W)}{83.9}$. wobci 39.3 und 83.9 den Glukosegehait. bzw. den Wert $(100-W)$ der Kontrolle bedeuten.

(2) Nicht angeimpfte, 14 Monate in umgebender Temperatur gelagerte Proben.

(3) Angeimpfte Probe vor der Lagerung.

peut paraitre paradoxal. Nous allons tenter d'expliquer cette anomalie. Pour cela, il convient de faire l'inventaire des principaux facteurs qui déterminent la cristallisation et de situer leur importance relative suivant les conditions. Une analyse plus détaillée du phénomène de cristallisation, avec application au cas du miel, est présentée dans une synthèse bibliographique sur la cristallisation des trois sucres : saccharose, lactose et dextrose que nous publions par ailleurs (TABOURET, 1978).

\section{Principaux facteurs de la cristallisation}

Le premier facteur important de la cristallisation est la sursaturation. La sursaturation mesure le gradient, c'est-à-dire le déséquilibre de concentration entre la solution actuelle et la solution juste saturée correspondante. La solution juste saturée de dextro- 
se pur à $20^{\circ} \mathrm{C}$ correspond à $D / W=0,90$ (VASATKo et SMELIK, 1967). La présence des autres sucres ne modifie que légèrement cette valeur de référence (JACKSON et SiLsBE, 1924). Par conséquent, tous les miels naturels sont très sursaturés en dextrose et devraient cristalliser rapidement, et d'autant plus rapidement que $D / W$ est plus élevé. Il n'en est rien. Toutefois, pour les miels courants de $W$ supérieure à 15 p. cent, les chances de cristallisation augmentent avec $D / W$. C'est pourquoi le rapport $D / W$ continue à servir d'indice de tendance à la cristallisation. Mais il ne représente qu imparfaitement la sursaturation et la sursaturation n'est pas le seul facteur à considérer.

Le deuxième facteur important de la cristallisation est le facteur " catalytique ". Le déséquilibre décrit précédemment peut amener une évolution déclenchée par un certain nombre de facteurs de catalyse plus ou moins bien connus : présence de cristaux (de dextrose ou autres), de pollen, de poussières, causes dynamiques telles que choc, agitation, choc thermique, etc. La pasteurisation protège le miel de façon relativement efficace contre ces facteurs, notamment contre l'ensemencement par des cristaux déjà pré sents au moment de la récolte, mais elle ne protège pas contre les causes qui peuvent survenir postérieurement à son application et, en fait, les miels qui ont une forte tendance à cristalliser finissent par recristalliser après pasteurisation, mais plus ou moins lentement.

Le troisième facteur de la cristallisation est la viscosité. Les échantillons déshydratés sont visiblement d'autant plus visqueux que moins riches en eau. Nous avons pu mesurer pour le même miel les viscosités suivantes à $40^{\circ} \mathrm{C}$ sur appareil RHEOMAT 15 de Contraves:

Miel de $W=16,1$ p. cent : 18 Poises.

Miel de $W=11,4$ p. cent : 118 Poises.

Des mesures de viscosité sur des miels de teneur en eau aussi basse n'ont jamais été rapportées dans la bibliographie.

Lorsque la viscosité est élevée, la migration des molécules de sucre dans la phase liquide vers les cristaux est ralentie et la cristallisation est, elle aussi, ralentie. C'est la raison pour laquelle nos échantillons déshydratés ont été conservés à température ambiante $\left(19\right.$ à $25^{\circ} \mathrm{C}$ ) plutôt qu'à $14^{\circ} \mathrm{C}$ qui est la température habituellement recommandée pour favoriser la cristallisation du miel; pour les échantillons déshydratés, la température ambiante est plus favorable à la cristallisation.

Mais l'explication n'est pas si simple. On sait, en effet, que certaines substances augmentent considérablement la viscosité des solutions de sucres sans agir nécessairement sur la vitesse de cristallisation. Ainsi, Codounis (1962) a montré que l'adjonction au miel d'alginates, de dextrines ou d'acides galacturoniques augmente sa viscosité mais ne diminue pas sa cristallisation. Il faut admettre ici que la structure de la solution ou du gel intervient : la structure réticulée due aux alginates et dextrines ne gêne pas la migration des molécules des sucres et ne gêne donc pas la cristallisation de ceux-ci. 
Le quatrième facteur important de la cristallisation est la composition de la solution. A côté du dextrose qui est le seul sucre susceptible de cristalliser, se trouvent en solution dans le miel d'autres sucres et des substances diverses appelées " non sucres ". Le lévulose se trouve en grande quantité. Il modifie peu la saturation du dextrose mais il abaisse considérablement la vitesse de cristallisation du dextrose : CoDounis (1962) a trouvé les résultats suivants :

- Une solution de composition $D>34 \quad L \leqslant 50 \quad$ Eau $=16$, soit $D / W>2,12$, cristallise en moins de 30 jours.

- Une solution de composition $D=32 \quad L=52 \quad$ Eau $=16$, soit $D / W=2$, cristallise à partir de 120 jours.

- Une solution de composition $D<28 \quad L \geqslant 56 \quad$ Eau $=16$, soit $D / W<1,75$, ne cristallise pas après un an.

Ces résultats confirment deux de JAMIESON (1954) obtenus en opérant directement sur du miel. Cette influence très marquée est la raison pour laquelle pendant très longtemps, et encore à l'heure actuelle, la tendance du miel à cristalliser a été comparée au rapport $D / L$.

Or, cette influence du lévulose reste inexpliquée. Elle ne peut être due à la seule viscosité : celle-ci croît avec la longueur des molécules de sucre. Le lévulose a sensiblement la même influence sur la viscosité que le dextrose et son influence sur la viscosité est moins marquee que celle des disacharides et bien moins que celle des polysaccharides. Pourtant les polysaccharides entravent bien moins la cristallisation que le lévulose. Le lévulose retarde aussi la cristallisation du saccharose, dans les produits de confiserie par exemple. D'autre part, il est très hygroscopique et très difficile à cristalliser.

Tous ces faits nous ont conduit à émettre l'hypothèse fondamentale suivante : le lévulose immobilise par solvatation les molécules d'eau et freine ainsi la migration des molécules de sucre, dextrose dans le cas du miel, saccharose dans le cas des confiseries, et il freine par là même la cristallisation de ces sucres en solution aqueuse. La solvation du dextrose est moins importante toutes choses égales d'ailleurs, comme l'ont montré tout récemment LoNCIN et al. (1978). Il revient au même de dire que le dextrose est moins hygroscopique que le lévulose. Par conséquent, le rôle inhibiteur de cristallisation du dextrose est moins marqué. Celui des sucres supérieurs est encore moindre, par exemple dans les sirops de glucose à bas dextrose équivalent, sauf pour les sucres à molécules très ramifiées. Partant de cette hypothèse, il est commode de faire intervenir un coefficient jusqu'ici bien peu mentionné dans la littérature apicole: l'activité de l'eau.

\section{Rôle de l'activité de l'eau}

L'activité de l'eau $a_{w}$ dans un produit solide ou liquide, dont nous avons précédemment rappelé la définition, est mesurée par un nombre compris entre 0 et 1 reflétant le 
degré de liberté de l'eau qu'il contient. Elle augmente avec la teneur en eau $W$ du produit et varie avec la température. D'autre part, la nature propre du produit est déterminante, bien plus encore que la teneur en eau de ce produit. C'est ainsi, par exemple, qu'un morceau de sucre (saccharose) à $W=0,5 \mathrm{p}$. cent est très " humide " en ce sens que son eau est disponible, pour une fermentation par exemple, car son activité de l'eau est voisine de 1 , alors qu'une solution saturée de lévulose à $20^{\circ} \mathrm{C}$, contenant $21,2 \mathrm{p}$. cent d'eau est très hygroscopique car son activité de l'eau est de 0,63 seulement (Herss, 1968) et ne peut fermenter.

Il est très intéressant de faire intervenir cette grandeur dans le cas du miel car elle tient compte à la fois de la teneur en eau $W$ du miel et du rôle inhibiteur de cristallisation du lévulose et accessoirement d'autres solutés (sucres surtout), c'est-à-dire de la disponibilité de l'eau, disponibilité qui conditionne dans une large mesure la cristallisation.

\section{Recherche d'un indice de la tendance à cristalliser}

A ce jour, un certain nombre d'indices ont été proposés. Rappelons-en quelquesuns :

- La sursaturation $\left({ }^{*}\right)$, exprimée en $\frac{\text { Dextrose p. cent }}{\text { Dextrose p. cent à saturation }}$

- Le rapport $\frac{D-W}{L}($ JACKSON et SiLSBEE, 1924).

- Le rapport $\frac{D}{W}$ (usuel).

- Le rapport $\frac{B-D}{D} \quad$ où $\quad B=100-W$ (Brix) (Codounis, 1962).

Dans ce dernier cas l'auteur fait intervenir le $p \mathrm{H}$ du miel.

Aucun de ces indices ne donne satisfaction (HADORN et ZürCHER, 1974).

Aucun indice ne peut rendre compte du facteur "catalytique " mentionné précédemment. L'influence des facteurs autres que catalytiques est d'autant mieux prise en compte par les indices que l'on considère une plage de teneurs en eau plus étroite et plus proche de la moyenne courante. En introduisant $a_{w}$ dans la détermination d'un indice on prend aussi en compte l'influence de $W$.

(*) Il y a de nombreuses façons différentes et non équivalentes d'exprimer la sursaturation (TABOURET, 1978). 
En examinant un certain nombre de publications nous avons pu, dans bien des cas, expliquer des anomalies apparentes par rapport au tableau 1 en faisant intervenir $a_{w}$ en même temps que $D / W$. Il est certes difficile de trouver une formulation mathématique satisfaisante pour tous les cas car les facteurs aléatoires restent nombreux. Nous proposons toutefois une formule empirique de la forme

$$
I_{n}=\frac{D / W}{\left(1-a_{w}\right)^{n}}
$$

où : $n=1$ à 1,5 pour les valeurs de $W$ supérieures à $17 \mathrm{p}$. cent et où $n=2$ pour les valeurs de $W$ inférieures à $17 \mathrm{p}$. cent.

Ces valeurs de $n$ ont été déterminées de façon empirique.

Examinons quelques exemples d'application de la formule que nous proposons.

Premier exemple. - Dans le tableau 5 sont reportées les principales données relatives aux miels de colza déshydratés extraites du tableau 3, ainsi que les valeurs de $I_{2}=\frac{D / W}{\left(1-a_{w}\right)^{2}}$. On constate que l'indice $I_{2}$ présente une bonne corrélation avec les résultats de cristallisation alors que l'indice $D / W$ est totalement en désaccord avec ces résultats : d'après le tableau 1 , en effet, tous les échantillons devraient avoir la note 9 ou en tous cas une note élevée et croissant avec $D / W$, alors qu'au contraire trois échantillons sur six au moins ont la note 0 et que les notes décroissent lorsque $D / W$ croît.

Deuxième exemple. - En utilisant les résultats trouvés par WHITE et al. (1962), nous avons établi le tableau 6 où figurent les quatre miels mentionnés par ces auteurs comme ayant une teneur en eau $W$ inférieure à $14 \mathrm{p}$. cent et la moyenne de tous les miels étudiés dans cette publication (cette moyenne se trouve page $121 \mathrm{de}$ ladite publication). A partir de l'analyse détaillée fournie pour chaque échantillon, nous avons calculé $a_{w}$ comme indiqué pages $5-6$ et $I_{2}=\frac{D / W}{\left(1-a_{w}\right)^{2}}$.

Si l'on compare la moyenne des quatre miels de faible $W$ à la moyenne de tous les miels, on constate que l'indice $D / W$ est en contradiction avec les résultats de cristallisation : d'après le tableau 1 , les miels de faible $W$ devraient avoir en moyenne la note 8 qui correspond à $D / W=2,16$, alors qu'ils ont la note 1 . En tous cas, ces miels devraient avoir une note supérieure à celle de la moyenne de tous les miels, c'est-à-dire à 3 , ce qui n'est pas le cas. Au contraire, les indices $I_{2}$ sont bien conformes aux résultats de cristallisation puisque $I_{2}$ des miels pauvres en eau est nettement inférieur à la moyenne générale de $I_{2}$.

Troisième exemple. - Le tableau 29 de la publication de WHITE et al. (1962) donne la composition moyenne des miels suivant leur origine florale et la note obtenue 
TABL. 6. - Cristallisation moyenne comparée des miels peu humides

et des miels d'humidité courante d'après WHITE et al. 1962.

ТАВ. 6. - Vergleich der mittleren Kristallisation von wasserarmen Honigen und Honigen mit normalem Wassergehalt nach WHITE et al. 1962.

\begin{tabular}{|c|c|c|c|c|c|c|}
\hline & \multicolumn{6}{|c|}{$\begin{array}{l}\text { Échantillon } \mathrm{n}^{\circ} \\
\text { Probe } \mathrm{n}^{\circ}\end{array}$} \\
\hline & 423 & 440 & 450 & 493 & $\begin{array}{c}\text { Moyenne } \\
\text { des précédents } \\
\text { Mittelwert } \\
\text { der } \\
\text { vorangegangenen } \\
\text { Zahlen }\end{array}$ & $\begin{array}{c}\begin{array}{c}\text { Moyenne } \\
\text { des }\end{array} \\
504 \text { échantillons } \\
\text { Mittelwert } \\
\text { der } \\
504 \text { Proben }\end{array}$ \\
\hline$W$ pour cent & 13,7 & 13,4 & 13,8 & 12,2 & 13,275 & 17,2 \\
\hline$D / W$ & 2,26 & 2,21 & 2,27 & 1,91 & 2,16 & 1,81 \\
\hline $\begin{array}{l}\text { Note de cristallisation }\left(^{*}\right) \\
\text { Benotung der Kristallisation }\end{array}$ & 1 & 1 & 1 & 1 & 1 & 3 \\
\hline $\begin{array}{l}a_{w} \text { calculée } \\
a_{n} \text {, berechnet }\end{array}$ & 0,545 & 0,543 & 0,539 & 0,536 & 0,539 & 0,612 \\
\hline$I_{2}=\frac{D / W}{\left(1-a_{w}\right)^{2}}$ & 10,92 & 10,60 & 10,68 & 8,87 & 10,175 & 12,043 \\
\hline
\end{tabular}

(*) Voir Tableau 1.

Siehe Tabelle 1.

lors de l'étude de la cristallisation (Voir tabl. 1). A partir de ces données on peut établir le tableau 7 dans lequel les miels sont reclassés par ordre de cristallisation décroissante.

La tendancé à cristalliser doit s'évaluer en tenant compte à la fois de la note obte nue et de l'âge de l'échantillon. Ainsi le miel de Verbenacea est à cristallisation très rapide car la cristallisation s'accélère lorsque la surface des cristaux augmente et on peut présumer que la note serait de 8 ou 9 après dix mois. Son classement d'après l'indice $D / W$ est très anormal alors que son classement selon $I_{2}$ est normal.

De même, compte tenu de l'âge, les miels notés 4 sont classés en fait dans le tableau 7 par ordre de vitesse de cristallisation décroissante de haut en bas. On constate que cet ordre est bien celui du classement d'après $I_{2}$, à une exception près : le miel de Rutacea devrait être au-dessous du miel d'Oleacea. Au contraire, le classement d'après $D / W$ est tout à fait aberrant car il donne de haut en bas : Rutacea, Oleacea, Salicacea, Clethracea, Verbenacea.

Enfin, si l'on compare la moyenne de l'ensemble des miels notés 4 à la moyenne de l'ensemble des miels notés 3 , le classement d'après $D / W$ est contraire, une fois de plus, aux résultats expérimentaux alors que le classement d'après $I_{2}$ est conforme. 
TAB. 7. - Tendance à cristalliser des miels suivant leur origine florale d'après WHITE et al. 1962.

TAB. 7. - Kristallisationsneigung der Honige nach ihrer sortenmässigen Herkunft, WHITE et al. 1962.

\begin{tabular}{|c|c|c|c|c|c|}
\hline $\begin{array}{c}\text { Origine florale } \\
\text { Botanische Herkunft }\end{array}$ & $\begin{array}{c}\text { Note }\left(^{*}\right) \\
\text { Note }\left(^{*}\right)\end{array}$ & $\begin{array}{c}\text { Age } \\
\text { (mois) } \\
\text { Alter } \\
\text { (Monate) }\end{array}$ & $D / W$ & $\begin{array}{l}a_{w} \text { calculée } \\
a_{w} \text { berechnet }\end{array}$ & $I_{2}=\frac{D / W}{\left(1-a_{w}\right)^{2}}$ \\
\hline Tamaricacea & 9 & 12 & 2,735 & 0,539 & 12,87 \\
\hline Malvacea & 8 & 10 & 2,27 & 0,585 & 13,175 \\
\hline Cucurbitacea & 5 & 11 & 1,96 & 0,611 & 12,96 \\
\hline Verbenacea & 4 & 6 & 1,42 & 0,692 & 14,94 \\
\hline Salicacea & 4 & 10 & 1,82 & 0,619 & 12,51 \\
\hline Clethracea & 4 & 12 & 1,76 & 0,623 & 12,37 \\
\hline Rutacea & 4 & 17 & 1,91 & 0,591 & 11,39 \\
\hline Oleacea & 4 & 19 & 1,88 & 0,597 & 11,55 \\
\hline $\begin{array}{l}\text { Moyenne des " } 4 \text { " } \\
\text { Mittelwert der " } 4 \mathrm{er} \text { " }\end{array}$ & 4 & 12,8 & 1,73 & 0,627 & 12,55 \\
\hline $\begin{array}{l}\text { Moyenne des \& } 3 \text { " } \\
\text { Mittelwert der " 3er " }\end{array}$ & 3 & 13,8 & 1,83 & 0,613 & 12,20 \\
\hline $\begin{array}{l}\text { Moyenne des " } 2 \text { " } \\
\text { Mittelwert der " } 2 \mathrm{er} \text { " }\end{array}$ & 2 & 13,6 & 1,71 & 0,615 & 11,54 \\
\hline $\begin{array}{l}\text { Moyenne des " } 1 \text { " } \\
\text { Mittelwert der " ler" }\end{array}$ & 1 & 13,1 & 1,72 & 0,606 & 11,09 \\
\hline $\begin{array}{l}\text { Moyenne des « } 0 \text { " } \\
\text { Mittelwert der " Oer " }\end{array}$ & 0 & 14,2 & 1,51 & 0,629 & 10,94 \\
\hline
\end{tabular}

(*) Voir Tableau 1.

Siehe Tabelle 1.

\section{CONCLUSIONS}

Il et aléatoire de vouloir exprimer la tendance des miels à cristalliser à l'aide d'un indice unique calculé d'après leur composition, ceci en raison de la complexité du phénomène de cristallisation et notamment du rôle de catalyseur de cristallisation que peuvent jouer par exemple des poussières, des variations thermiques, etc.

Toutefois, dans la mesure où ces risques peuvent être réduits grâce à une pasteurisation convenable, on peut apprécier la tendance à cristalliser à partir des indices $D / W$, $D / L$ et $a_{w}$ considérés simultanément.

L'activité de l'eau $a_{w}$ peut être mesurée expérimentalement (voir page 345) mais la valeur approximative peut aussi être calculée à partir de la composition du miel (voir page 5). Son influence est d'autant plus grande que la teneur en eau $W$ du miel est plus basse. Elle permet d'expliquer de nombreux cas de cristallisation non conformes au 
tableau de WhiTE et al. (Voir tabl. 1). Lorsque la teneur en eau du miel est ramenée à 13 p. cent environ, la cristallisation est fortement inhibee même si le miel est ensemencé.

Reçu pour publication en avril 1979

Eingegangen im April 1979

\section{REMERCIEMENTS}

Nous tenons à remercier ici pour leur collaboration Mlle Courant, documentaliste à la Station de recherches sur l'Abeille et les Insectes sociaux de Bures-sur-Yvette, Mlle LABRoche, responsable du Laboratoire-Photo de l'I.U.T. de Dijon, ainsi que M. Louveaux, directeur de la Station de recherches de Bures-sur-Yvette et M. BorNECK, directeur de l'Institut technique d'Apiculture.

\section{ZUSAMMENFASSUNG}

Die Kennzahl von J. W. WhIte (1962) $\frac{D}{W}=\frac{\text { Glukose }}{\text { Wasser }}$ beschreibt die Kristallisationstendenz der Honige nur unzureichend. Besonders wasserarme Honige haben eine geringere Kristallisationsneigung, als nach dem Index $D / W$ zu erwarten wäre.

Die vorliegende Untersuchung befasste sich mit der Kristallisation wasserarmer Honige mit Wassergehalten zwischen acht Prozent und sechzehn Prozent und den entsprechenden Gleichgewichtsfeuchtigkeiten (Wasseraktivitäten). Die Wasseraktivität $\left(a_{w}\right)$ wird wie folgt definiert : $a_{w}=\frac{f_{t}}{F_{t}}$. Dabei bedeutet $f_{t}$ den Wasserdampfdruck an der Oberfläche des Produkts, in unserem Falle des Honigs und $F_{t}$ den Wasserdampfdruck über einer freien Wasserfläche der gleichen Temperatur. Die Wasseraktivität eines Honigs ändert sich gegenläufig zu seiner Wasseranziehung (Hygroskopie).

Die verschiedenen Muster wurden aus einem Rapshonig hergestellt. Dieser wurde zuerst unter Vakuum getrocknet und danach mit Wasser stufenweise verdünnt.

Bei jeder Probe wurden die Gleichgewichtsfeuchtigkeit $a_{w}$ gemessen und die Entwicklung der Kristallisation photographisch dokumentiert.

Dies geschah bei Mustern, die vorher mit Glukosekristallen angeimpft worden waren und bei nicht geimpften Mustern.

Muster mit Wassergehalten unter $13 \%$ zeigen eine sehr geringe Kristallisationstendenz, selbst wenn sie vorher angeimpft worden waren. Es wurde eine zufrieden stellende Wechselbeziehung zwischen ihrer Kristallisationstendenz und dem folgenden Index ermittelt :

$$
I_{2}=\frac{D / W}{\left(1-a_{w}\right)^{2}}
$$

Eine ähnliche Korrelation wurde für frühere Arbeiten, besonders für WHITE (1962) gefunden, nach der Gleichung :

$$
I_{n}=\frac{D / W}{\left(1-a_{w}\right)^{n}}
$$


wobei $n$ mit der Trockensubstanz in Prozent steigt und $a_{w}$ nach der Methode von Grover berechnet werden kann. Eine Erklärung über die Rolle der Fruktose wird diskutiert. Einige Faktoren, die die Kristallisationsentwicklung wesentlich beeinflussen, aber nicht mit der Zusammensetzung des Honigs zusammenhängen, wie z.B. eine Animpfung, Staub usw. lassen sich nicht in Kennzahlen fassen.

\section{BIBLIOGRAPHIE}

A.S.T.M. - (American Society for Testing and Materials). 1951-1963. 1916 Race Street Philadelphia, Pa 19103. Recommended Practice E 104-51.

Austin G. H., 1956. - Maltose Content of Canadian honeys and its probable effects on crystallisation. Proceedings tenth international congress of entomology. Vol. 4, 1001-1006.

Borneck R., Tabouret T., 1977. - Déshydratation du miel sur centritherm. Apiacta, 12 (4) : 174-175.

Codounis M., 1962. - La cristallisation du miel (traduit du grec). Institut de technologie végétale, Ministère de l'Agriculture; Athènes, $88 \mathrm{p}$.

Crane E., 1975. - Honey. A comprehensive survey, London, Heinemann, 608 p.

FULmer et BosCH, 1932 et 1934. - Cités par Jamieson, 1954.

Gonnet M., 1977. - Refonte, pasteurisation et cristallisation dirigée du miel. Apiacta, 12 (3) : $105-109$.

Grover D. W., 1947. - Keeping Properties of confectionary. J. Soc. chem. Ind., 66, 201-205.

HAdORN H., ZÜRCHER K., 1974. - Zuckerspecktrum und Kristallisationstendenz von Honigen. Mitt. Gebiete Lebensm. Hyg. 65, 407-420.

HeIss R., 1968. - Haltbarkeit und Sorptionsverhalten wasserarmer Lebensmittel. Springer Verlag Berlin, Heidelberg, New York, 163.

JACKSON R. F., SILsbeE C. G., 1924. - Saturation relations in mixtures of sucrose, dextrose and levulose. Technol. Papers of Bureau of Standards, Washington DC, vol. 18, 277-304.

JAMIESON C. A., 1954. - Some factors influencing the crystallization of honey. Annual report of the state Apiarist. State of Iowa, 64-73.

Loncin H., Weisser H., Audu K., 1978. - Sorption isotherms of sugars. Lebensm. Wiss. u. Technol. 11, 31-34.

Martin, 1958. - Cité par E. Crane, 1975.

Money R. W., Born R., 1951. - Equilibrium humidity of sugar solutions. J. Sci. Food Agric., april 180-185.

NoRRISH R. S., 1966. - An equation for the activity coefficients and equilibrium relative humidities of water in confectionary syrups. J. Food Technol. 1, 25-39.

Siddiqui I. R., 1970. - The sugars of honey. Adv. Carbohydrate Chem. and Biochem., 25, $285-309$.

TABouret T., 1978. - Technologie de la cristallisation. Aspects théoriques et pratiques dans quelques industries alimentaires. Synthèse bibliographique 80 p. 150 références. Diffusion par le Centre de Documentation des Industries Utilisatrices de Produits Agricoles. Avenue des Olympiades, 91305 Massy.

TABouret T., 1977. - Essais de déshydratation sous vide du miel, Apiacta, 12 (4) : 157-164.

Vasatko J., Smelik A., 6 juin 1967. - Die Kristallisation der Saccharose, D Glukose und D Fruktose aus übersättigten Lösungen des metastabilen Gebiets. Communication à la $13^{\mathrm{c}}$ assemblée de la Commission Internationale des Techniciens de Sucrerie à Falsterbo (Suède), p. 69-96. Secrétariat Général de la C.I.T.S., 1 Aandoren Straat. Tienen, Tirlemont (Belgique).

White J. W., Riethof M. L., Subers M. H., Kushnir T., 1962. - Composition of american honeys. Eastern utilization research and development division, Agricultural Research Service. Philadelphia Pa. Technical Bulletin $n^{\circ} 1261,124$ p. Government printing Office. Washington 25, DC. 